EUROPEAN JOURNAL OF PURE AND APPLIED MATHEMATICS

Vol. 11, No. 1, 2018, 35-50

ISSN 1307-5543 - www.ejpam.com

Published by New York Business Global

\title{
On Generalizations of $\phi$-2-absorbing primary submodules
}

\author{
Pairote Yiarayong ${ }^{1, *}$, Manoj Siripitukdet ${ }^{1}$ \\ 1 Department of Mathematics, Faculty of Science, Naresuan University, \\ Phitsanuloke 65000, Thailand
}

\begin{abstract}
Let $\phi: S(M) \rightarrow S(M) \cup\{\emptyset\}$ be a function where $S(M)$ is the set of all submodules of $M$. In this paper, we extend the concept of $\phi$-2-absorbing primary submodules to the context of $\phi$-2-absorbing semi-primary submodules. A proper submodule $N$ of $M$ is called a $\phi$-2-absorbing semi-primary submodule, if for each $m \in M$ and $a_{1}, a_{2} \in R$ with $a_{1} a_{2} m \in N-\phi(N)$, then $a_{1} a_{2} \in \sqrt{(N: M)}$ or $a_{1} m \in N$ or $a_{2}^{n} m \in N$ for some positive integer $n$. Those are extended from 2 -absorbing primary, weakly 2 -absorbing primary, almost 2 -absorbing primary, $\phi_{n}$-2-absorbing primary, $\omega$-2-absorbing primary and $\phi$-2-absorbing primary submodules, respectively. Some characterizations of 2-absorbing semi-primary, $\phi_{n}$-2-absorbing semi-primary and $\phi$-2-absorbing semiprimary submodules are obtained. Moreover, we investigate relationships between 2-absorbing semi-primary, $\phi_{n}$-2-absorbing semi-primary and $\phi$-primary submodules of modules over commutative rings. Finally, we obtain necessary and sufficient conditions of a $\phi$-2-absorbing semi-primary in order to be a 2-absorbing semi-primary submodule.
\end{abstract}

2010 Mathematics Subject Classifications: 13C05, 13C13.

Key Words and Phrases: 2-absorbing semi-primary submodule, $\phi_{\alpha}$-2-absorbing semi-primary submodule, $\phi$-2-absorbing primary submodule, $\phi$-primary ideal, $\phi$-2-absorbing ideal.

\section{Introduction}

Throughout this paper, we assume that all rings are commutative with a nonzero identity Suppose that $R$ is a ring and $M$ is an $R$-module The concept of $\varphi$-prime ideals, a generalization of prime ideals was introduced and investigated in [2]. Let $\varphi: \mathcal{J}(R) \rightarrow$ $\mathcal{J}(R) \cup\{\emptyset\}$ be a function where $\mathcal{J}(R)$ is a set of ideals of $R$. A proper ideal $I$ of $R$ is said to be $\varphi$-prime if whenever $a_{1}, a_{2} \in R$ and $a_{1} a_{2} \in I-\varphi(I)$, then $a_{1} \in I$ or $a_{2} \in I$. Since $I-\varphi(I)=I-(I \cap \varphi(I))$, so without loss of generality, throughout this paper we will consider $\varphi(I) \subseteq I$. Later, Darani [7] gave a generalization of primary ideals which covers all the above mentioned definitions. He defined the a proper ideal $I$ of $R$ is said to be $\varphi$-primary if for $a_{1}, a_{2} \in R$ with $a_{1} a_{2} \in I-\varphi(I)$, either $a_{1} \in I$ or $a_{2}^{n} \in I$ for some

${ }^{*}$ Corresponding author.

Email addresses: pairote0027@hotmail.com (Pai. Yiarayong), manojs@nu.ac.th (M. Siripitukdet) 
positive integer $n$. Thus a $\varphi$-prime ideal is just a $\varphi$-primary ideal. In [9], Ebrahimpour and Nekooei called a proper ideal $I$ of a commutative ring $R$ to be $\varphi$-2-absorbing if whenever $a_{1}, a_{2}, a_{3} \in R$ and $a_{1} a_{2} a_{3} \in I-\varphi(I)$, either $a_{1} a_{2} \in I$ or $a_{2} a_{3} \in I$ or $a_{1} a_{3} \in I$. Badawi, et al. [1] generalized the concept of 2-absorbing primary ideals to $\varphi$-2-absorbing primary ideals. According to their definition, a proper ideal $I$ of $R$ is called a $\varphi$-2-absorbing primary ideal if whenever $a_{1} a_{2} a_{3} \in I-\varphi(I)$ for $a_{1}, a_{2}, a_{3} \in R$, then $a_{1} a_{2} \in I$ or $a_{2} a_{3} \in \sqrt{I}$ or $a_{1} a_{3} \in \sqrt{I}$. Clearly a $\varphi$-2-absorbing ideal of $R$ is also a $\varphi$-2-absorbing primary ideal of $R$. Other generalizations of prime ideals have recently been studied in $[4,3,5,6]$.

The notion of $\phi$-prime submodule, which is a generalization of prime submodule, was introduced by Zamani in [11]. Let $\phi: S(M) \rightarrow S(M) \cup\{\emptyset\}$ be a function where $S(M)$ is a set of all submodules of $M$. A proper submodule $N$ of $M$ is called $\phi$-prime submodule of $M$ if whenever $a \in R$ and $a m \in N-\phi(N)$, then $m \in N$ or $a \in(N: M)$. Since $N-\phi(N)=N-(N \cap \phi(I))$, without loss of generality we may assume that $\phi(N) \subseteq N$. Recall that a proper submodule $N$ of $M$ is called a $\phi$-primary submodule submodule of $M$ as in [11] if whenever $a m \in N-\phi(N)$ for some $a \in R, m \in M$, then $m \in N$ or $a^{n} \in(N: M)$ for some positive integer $n$. In 2017, Ebrahimpour and Mirzaee [8] generalized the concept of semiprime submodules to $\phi$-semiprime submodules. According to their definition, a proper submodule $N$ of $M$ is called a $\phi$-semiprime submodule if whenever $a^{2} m \in N-\phi(N)$ for $a \in R, m \in M$, then $a m \in N$. In [10], the concept of $\phi$-prime and $\phi$-primary submodules generalized to $\phi$-2-absorbing primary submodule of a module over a commutative ring. Let $N$ be a proper submodule of $M . N$ is said to be a $\phi$-2-absorbing primary submodule of $M$ if whenever $a_{1}, a_{2} \in R$ and $m \in M$ with $a_{1} a_{2} m \in N-\phi(N)$, then $a_{1} a_{2} \in \sqrt{(N: M)}$ or $a_{1} m \in N$ or $a_{2} m \in N$. Moreover, recall from [10] that a proper submodule $N$ of $M$ is said to be a $\phi$-2-absorbing submodule of $M$ if whenever $a_{1}, a_{2} \in R$ and $m \in M$ with $a_{1} a_{2} m \in N-\phi(N)$ implies $a_{1} a_{2} \in(N: M)$ or $a_{1} m \in N$ or $a_{2} m \in N$. Thus a $\phi$-2-absorbing submodule is just a $\phi$-2-absorbing primary submodule.

In this paper, we extend the concept of $\phi$-2-absorbing primary submodule to the context of $\phi$-2-absorbing semi-primary submodule. Let $\phi: S(M) \rightarrow S(M) \cup\{\emptyset\}$ be a function where $S(M)$ is the set of all submodules of $M$. A proper submodule $N$ of $M$ is called a $\phi$-2-absorbing semi-primary submodule if for each $m \in M$ and $a_{1}, a_{2} \in R$ with $a_{1} a_{2} m \in N-\phi(N)$, then $a_{1} a_{2} \in \sqrt{(N: M)}$ or $a_{1} m \in N$ or $a_{2}^{n} m \in N$ for some positive integer $n$. Let $N$ be a $\phi$-2-absorbing semi-primary submodule of $M$.

- If $\phi(N)=\emptyset$ for every $N \in \mathcal{S}(M)$, then we say that $\phi=\phi_{\emptyset}$ and $N$ is called a $\phi_{\emptyset}-2-$ absorbing semi-primary submodule of $M$, and hence $N$ is a 2-absorbing semi-primary submodule of $M$.

- If $\phi(N)=\{0\}$ for every $N \in \mathcal{S}(M)$, then we say that $\phi=\phi_{0}$ and $N$ is called a $\phi_{0}$-2-absorbing semi-primary submodule of $M$, and hence $N$ is a weakly 2-absorbing semi-primary submodule of $M$.

- If $\phi(N)=N$ for every $N \in \mathcal{S}(M)$, then we say that $\phi=\phi_{1}$ and $N$ is called a $\phi_{1}$-2-absorbing semi-primary submodule of $M$. It is easy to see that every proper 
submodule is $\phi_{1}$-2-absorbing semi-primary.

- If $\phi(N)=(N: M) N$ for every $N \in \mathcal{S}(M)$, then we say that $\phi=\phi_{2}$ and $N$ is called a $\phi_{2}$-2-absorbing semi-primary submodule of $M$, and hence $N$ is an almost 2-absorbing semi-primary submodule of $M$.

- If $\phi(N)=(N: M)^{n-1} N$ for every $N \in \mathcal{S}(M)$, then we say that $\phi=\phi_{n \geq 2}$ and $N$ is called a $\phi_{n}$-2-absorbing semi-primary submodule of $M$, and hence $N$ is a $n-2$ absorbing semi-primary submodule of $M$.

- If $\phi(N)=\bigcap_{i=1}^{\infty}(N: M)^{i} N$ for every $N \in \mathcal{S}(M)$, then we say that $\phi=\phi_{\omega}$ and $N$ is called a $\phi_{\omega}$-2-absorbing semi-primary submodule of $M$, and hence $N$ is a $\omega$-2absorbing semi-primary submodule of $M$.

In section 2 , we give some basic properties of $\phi$-2-absorbing semi-primary submodules. Among many results in this paper, it is shown that $N$ is a $\phi$-2-absorbing semi-primary submodule of $M$ if and only if for every $a_{1}, a_{2} \in R-(N: M)$ and $a_{1} a_{2} \in R-\sqrt{(N: M)}$, $\left(N: a_{1} a_{2}\right) \subseteq\left(\phi(N): a_{1} a_{2}\right) \cup\left(N: a_{1}\right) \cup\left(N: a_{2}^{n}\right)$ for some positive integer $n$.

In Section 3, we study the stability of $\phi_{\alpha}$-2-absorbing semi-primary submodules. Moreover, we investigate relationships between 2-absorbing semi-primary, $\phi_{0}$-2-absorbing semiprimary, $\phi_{n}$-2-absorbing semi-primary and $\phi$-primary submodules of modules over commutative rings. Finally, we obtain necessary and sufficient conditions of a $\phi$-2-absorbing semi-primary in order to be a 2 -absorbing semi-primary.

\section{Properties of $\phi$-2-Absorbing Semi-primary Submodules}

The results of the following theorems seem to play an important role to study $\phi$ classical semi-primary submodules of modules over commutative rings; these facts will be used frequently and normally we shall make no reference to this definition.

Definition 1. Let $M$ be an $R$-module and let $\phi: S(M) \rightarrow S(M) \cup\{\emptyset\}$ be a function where $S(M)$ be a set of all submodules of $M$. A proper submodule $N$ of $M$ is called a $\phi$-2-absorbing semi-primary submodule, if for each $m \in M$ and $a_{1}, a_{2} \in R$ with $a_{1} a_{2} m \in$ $N-\phi(N)$, then $a_{1} a_{2} \in \sqrt{(N: M)}$ or $a_{1} m \in N$ or $a_{2}^{n} m \in N$ for some positive integer $n$.

Remark 1. It is easy to see that every $\phi$-2-absorbing primary submodule is $\phi$-2-absorbing semi-primary.

The following example shows that the converse of Remark 1 is not true.

Example 1. Let $R=\boldsymbol{Z}$ and $M=\boldsymbol{Z}$. Consider the submodule $N=12 \boldsymbol{Z}$ of $M$. Define $\phi: S(M) \rightarrow S(M) \cup\{\emptyset\}$ by $\phi(N)=\{0\}$ for every $N \in S(M)$. It is easy to see that $N$ is a $\phi$-2-absorbing semi-primary submodule of $M$. Notice that $2 \cdot 2 \cdot 3 \in N-\phi(N)$, but $2 \cdot 3 \notin N$ and $(2 \cdot 2)^{n} \notin(N: M)$ for all positive integer $n$. Therefore $N$ is not a $\phi$-2-absorbing primary submodule of $M$. 
Theorem 1. Let $\phi: S(M) \rightarrow S(M) \cup\{\emptyset\}$ and $\varphi: \mathcal{J}(R) \rightarrow \mathcal{J}(R) \cup\{\emptyset\}$ be two functions.

(i) If $N$ is a $\phi$-2-absorbing semi-primary submodule of $M$, then $(N: m)$ is a $\varphi$-2absorbing primary ideal of $R$ with $m \in M-N$ and $(\phi(N): m)) \subseteq \varphi(N: m)$.

(ii) For every $m \in M-N$ if $(N: m)$ is a $\varphi$-primary ideal of $R$, then $N$ is a $\phi$-2-absorbing semi-primary submodule of $M$ with $\varphi(N: m) \subseteq(\phi(N): m))$.

Proof. 1. Let $a_{1}, a_{2}, a_{3} \in R$ such that $a_{1} a_{2} a_{3} \in(N: m)-\varphi((N: m))$. By assumption, $a_{1} a_{3}\left(a_{2} m\right) \in N-\phi(N)$. Then by Definition $1, a_{1} a_{3} \in \sqrt{(N: M)} \subseteq \sqrt{(N: m)}$ or $a_{1} a_{2} m \in$ $N$ or $a_{3}^{n} a_{2} m \in N$ for some positive integer $n$. Therefore $a_{1} a_{2} \in(N: m)$ or $a_{2} a_{3} \in$ $\sqrt{(N: m)}$ or $a_{1} a_{3} \in \sqrt{(N: m)}$. This completes the proof.

2. Let $a_{1}, a_{2} \in R$ such that $a_{1} a_{2} m \in N-\phi(N)$. Then $a_{1} a_{2} \in(N: m)$ and $a_{1} a_{2} \notin$ $(\phi(N): m)$. By assumption, $a_{1} a_{2} \in(N: m)-\varphi((N: m))$. Again, by assumption, $a_{1} \in(N: m)$ or $a_{2}^{n} \in(N: m)$ for some positive integer $n$. This completes the proof.

The following example shows that the converse of Theorem 1 is not true.

Example 2. 1. Let $M=\boldsymbol{Z} \times \boldsymbol{Z} \times \boldsymbol{Z}$ be an $\boldsymbol{Z}$-module. Define $\varphi: \mathcal{J}(R) \rightarrow \mathcal{J}(R) \cup\{\emptyset\}$ by $\varphi(I)=\{0\}$ for every $I \in \mathcal{J}(R)$. Consider the submodule $N=\{0\} \times 12 \boldsymbol{Z} \times \boldsymbol{Z}$ of M. Clearly, $\left(N:\left(m_{1}, m_{2}, m_{3}\right)\right)=\{0\}$ is a $\varphi$-2-absorbing primary ideal of $\mathcal{J}(R)$, where $\left(m_{1}, m_{2}, m_{3}\right) \in M-N$. Define $\phi: S(M) \rightarrow S(M) \cup\{\emptyset\}$ by $\phi(N)=\{(0,0,0)\}$ for every $N \in S(M)$. Notice that $3 \cdot 4(0,1,1) \in N-\phi(N)$, but $(3 \cdot 4) \notin \sqrt{(N: M)}, 3(0,1,1) \notin N$ and $4^{n}(0,1,1) \notin N$ for all positive integer $n$. Hence $N$ is not a $\phi$-2-absorbing semi-primary submodule of $M$.

2. Let $M=\boldsymbol{Z}_{12}$ be an $\boldsymbol{Z}_{12}$-module. Define $\phi: S(M) \rightarrow S(M) \cup\{\emptyset\}$ by $\phi(N)=\{[0]\}$ for every $N \in S(M)$. Consider the submodule $N=\{[0]\}$ of $M$. Clearly, $N$ is a $\phi$-2-absorbing semi-primary submodule of $M$. Define $\varphi: \mathcal{J}(R) \rightarrow \mathcal{J}(R) \cup\{\emptyset\}$ by $\varphi(I)=\emptyset$ for every $I \in \mathcal{J}(R)$. Notice that $[4][3] \in\{[0]\}=(N:[1])-\varphi((N:[1]))$, but $[4] \in(N:[1])$ and $[3]^{n} \in(N:[1])$ for all positive integer $n$.

Let $N$ be a submodule of an $R$-module $M$ and let $\phi: S(M) \rightarrow S(M) \cup\{\emptyset\}$ be a function. Define $\phi_{N}: S(M / N) \rightarrow S(M / N) \cup\{\emptyset\}$ by

$$
\phi_{N}(K / N)= \begin{cases}(\phi(K)+N) / N & ; \phi(K) \neq \emptyset \\ \emptyset & ; \phi(K)=\emptyset\end{cases}
$$

for every submodule $K$ of $M$ with $N \subseteq K$ [11]. In, 2010 Zamani in [11] gives relations between $\phi$-prime submodules of $M$ and $\phi_{N}$-prime submodules of $M / N$. This leads us to give relations between $\phi$-2-absorbing semi-primary submodules of $M$ and $\phi_{N}$-2-absorbing semi-primary submodules of $M / N$.

Theorem 2. Let $\phi: S(M) \rightarrow S(M) \cup\{\emptyset\}$ be a function and let $N, K$ be two submodules of $M$ with $N \subseteq K$. If $K$ is a $\phi$-2-absorbing semi-primary submodule of $M$, then $K / N$ is a $\phi_{N}$-2-absorbing semi-primary submodule of $M / N$. 
Proof. Let $a_{1}, a_{2} \in R$ and $m \in M$ such that $a_{1} a_{2}(m+N) \in(K / N)-\phi_{N}(K / N)$. Then $a_{1} a_{2} m \in K-\phi(K)$. By Definition 1, $a_{1} a_{2} \in \sqrt{(K: M)}$ or $a_{1} m \in K$ or $a_{2}^{n} m \in K$ for some positive integer $n$. Clearly, $a_{1} a_{2} \in \sqrt{(K / N: M / N)}$ or $a_{1}(m+N) \in K / N$ or $a_{2}^{n}(m+N) \in K / N$ for some positive integer $n$. This completes the proof.

Theorem 3. Let $\phi: S(M) \rightarrow S(M) \cup\{\emptyset\}$ be a function and let $N, K$ be two submodules of $M$. If $N \subseteq \phi(K)$ and $K / N$ is a $\phi_{N}-2$-absorbing semi-primary submodule of $M / N$, then $K$ is a $\phi$-2-absorbing semi-primary submodule of $M$.

Proof. Let $a_{1}, a_{2} \in R$ and $m \in M$ such that $a_{1} a_{2} m \in K-\phi(K)$. Then $a_{1} a_{2}(m+N) \in$ $(K-\phi(K)) / N$. By Definition 1, $a_{1} a_{2} \in \sqrt{(K / N: M / N)}$ or $a_{1}(m+N) \in K / N$ or $a_{2}^{n}(m+N) \in K / N$ for some positive integer $n$. Clearly, $a_{1} a_{2} \in \sqrt{(K: M)}$ or $a_{1} m \in K$ or $a_{2}^{n} m \in K$ for some positive integer $n$.

Now, by Theorem 2 and Theorem 3, we have the following corollary.

Corollary 1. Let $\phi: S(M) \rightarrow S(M) \cup\{\emptyset\}$ be a function and let $N, K$ be two submodules of $M$ with $N \subseteq \phi(K)$. Then $K$ is a $\phi$-2-absorbing semi-primary submodule of $M$ if and only if $K / N$ is a $\phi_{N}$-2-absorbing semi-primary submodule of $M / N$.

Proof. The proof follows from Theorem 2, 3 .

Zamani in [11] gives relations between $\phi$-prime submodules of $M$ and $\phi_{S}$-prime submodules of $S^{-1} M$. This leads us to give relations between $\phi$-2-absorbing semi-primary submodules of $M$ and $\phi_{S^{-}}$-absorbing semi-primary submodules of $S^{-1} M$.

Theorem 4. Let $S$ be a multiplicative closed subset of $R$ and let $\phi: S(M) \rightarrow S(M) \cup\{\emptyset\}$ be a function. If $N$ is a $\phi$-2-absorbing semi-primary submodule of $M$, then $S^{-1} N$ is a $\phi_{S}$-2-absorbing semi-primary submodule of $S^{-1} M$.

Proof. Let $a_{1}, a_{2} \in R, s_{1}, s_{2}, s_{3} \in S$ and $m \in M$ such that $\frac{a_{1}}{s_{1}} \frac{a_{2}}{s_{2}} \frac{m}{s_{3}} \in S^{-1} N-\phi_{S}\left(S^{-1} N\right)$. Then there exists $s \in S$ such that $s a_{1} a_{2} m \in N$. If $s a_{1} a_{2} m \in \phi(N)$, then $\frac{a_{1}}{s_{1}} \frac{a_{2}}{s_{2}} \frac{m}{s_{3}}=\frac{s a_{1} a_{2} m}{s s_{1} s_{2} s_{3}} \in$ $S^{-1} \phi(N)=\phi_{S}\left(S^{-1} N\right)$, a contradiction. Now if $s a_{1} a_{2} m \notin \phi(N)$, then $a_{1} a_{2}(s m) \in N-$ $\phi(N)$. By Definition $1, a_{1} a_{2} \in \sqrt{(N: M)}$ or $a_{1} s m \in N$ or $a_{2}^{n} s m \in N$ for some positive integer $n$. If $a_{1} s m \in N$ or $a_{2}^{n} s m \in N$, then $\frac{a_{1}}{s_{1}} \frac{m}{s_{3}}=\frac{a_{1} s m}{s_{1} s s_{3}} \in S^{-1} N$ or $\left(\frac{a_{2}}{s_{2}}\right)^{n} \frac{m}{s_{3}}=\frac{a_{2}^{n} s m}{s_{2}^{n} s_{3} s} \in$ $S^{-1} N$. Now if $a_{1} a_{2} \in \sqrt{(N: M)}$, then there exists positive integer $n_{1}$ such that $\left(a_{1} a_{2}\right)^{n_{1}} \in$ $(N: M)$. Clearly, $\left(\frac{a_{1}}{s_{1}} \frac{a_{2}}{s_{2}}\right)^{n} \in S^{-1}(N: M)$. This completes the proof.

Theorem 5. Let $S$ be a multiplicative closed subset of $R$ and let $\phi: S(M) \rightarrow S(M) \cup\{\emptyset\}$ be a function. If $S^{-1} N$ is a $\phi_{S}$-2-absorbing semi-primary submodule of $S^{-1} M$ such that $S \cap Z d(N / \phi(N))=\emptyset$ and $S \cap Z d(M / N)=\emptyset$, then $N$ is a $\phi$-2-absorbing semi-primary submodule of $M$.

Proof. Let $a_{1}, a_{2} \in R$ and $m \in M$ such that $a_{1} a_{2} m \in N-\phi(N)$. Then $\frac{a_{1}}{1} \frac{a_{2}}{1} \frac{m}{1}=\frac{a b m}{1} \in$ $S^{-1} N$. If $\frac{a_{1}}{1} \frac{a_{2}}{1} \frac{m}{1} \in \phi_{S}\left(S^{-1} N\right)=S^{-1} \phi(N)$, then there exists $s \in S$ such that $s a_{1} a_{2} m \in$ $\phi(N)$ which is a contradiction. If $\frac{a_{1}}{1} \frac{a_{2}}{1} \frac{m}{1} \notin \phi_{S}\left(S^{-1} N\right)$, then $\frac{a_{1}}{1} \frac{a_{2}}{1} \frac{m}{1} \in S^{-1} N-\phi_{S}\left(S^{-1} N\right)$. 
By Definition 1, $\frac{a_{1}}{1} \frac{a_{2}}{1} \in \sqrt{\left(S^{-1} N: S^{-1} M\right)}$ or $\frac{a_{1}}{1} \frac{m}{1} \in S^{-1} N$ or $\left(\frac{a_{2}}{1}\right)^{n} \frac{m}{1} \in S^{-1} N$ for some positive integer $n$. If $\frac{a_{1}}{1} \frac{a_{2}}{1} \in \sqrt{\left(S^{-1} N: S^{-1} M\right)}$, then $\left(\frac{a_{1}}{1} \frac{a_{2}}{1}\right)^{n} \in\left(S^{-1} N: S^{-1} M\right)$ for some positive integer $n$. Thus there exists $s \in S$ such that $s\left(a_{1} a_{2}\right)^{n} M \subseteq N$ for some positive integer $n$. By assumption, $\left(a_{1} a_{2}\right)^{n} M \subseteq N$ so $a_{1} a_{2} \in \sqrt{(N: M)}$.

In view of Theorem 4 and Theorem 5 , we have the following result.

Corollary 2. Let $S$ be a multiplicative closed subset of $R$ and let $\phi: S(M) \rightarrow S(M) \cup\{\emptyset\}$ be a function with $S \cap Z d(N / \phi(N))=\emptyset$ and $S \cap Z d(M / N)=\emptyset$. Then $N$ is a $\phi$-2-absorbing semi-primary submodule of $M$ if and only if $S^{-1} N$ is a $\phi_{S}$-2-absorbing semi-primary submodule of $S^{-1} M$.

Proof. The proof follows from Theorem 4, 5 .

In the following result, we give an equivalent definition of $\phi$-2-absorbing semi-primary submodules.

Theorem 6. Let $\phi: S(M) \rightarrow S(M) \cup\{\emptyset\}$ be a function. The following conditions are equivalent:

(i) $N$ is a $\phi$-2-absorbing semi-primary submodule of $M$.

(ii) For every $a_{1}, a_{2} \in R-(N: M)$ if $a_{1} a_{2} \in R-\sqrt{(N: M)}$, then $\left(N: a_{1} a_{2}\right) \subseteq(\phi(N)$ : $\left.a_{1} a_{2}\right) \cup\left(N: a_{1}\right) \cup\left(N: a_{2}^{n}\right)$ for some positive integer $n$.

Proof. $(i \Rightarrow i i)$ Let $m \in\left(N: a_{1} a_{2}\right)$. Then $a_{1} a_{2} m \in N$. If $a_{1} a_{2} m \in \phi(N)$, then $m \in\left(\phi(N): a_{1} a_{2}\right) \cup\left(N: a_{1}\right) \cup\left(N: a_{2}^{n}\right)$ for some positive integer $n$. If $a_{1} a_{2} m \notin \phi(N)$, then $a_{1} a_{2} m \in N-\phi(N)$. By Definition $1, a_{1} a_{2} \in \sqrt{(N: M)}$ or $a_{1} m \in N$ or $a_{2}^{n} m \in N$ for some positive integer $n$. By assumption, $m \in\left(N: a_{1}\right)$ or $m \in\left(N: a_{2}^{n}\right)$ for some positive integer $n$. Therefore $\left(N: a_{1} a_{2}\right)=\left(\phi(N): a_{1} a_{2}\right) \cup\left(N: a_{1}\right) \cup\left(N: a_{2}^{n}\right)$ for some positive integer $n$.

$(i i \Rightarrow i)$ It is obvious.

Corollary 3. Let $\phi: S(M) \rightarrow S(M) \cup\{\emptyset\}$ be a function. The following conditions are equivalent:

(i) $N$ is a $\phi$-2-absorbing semi-primary submodule of $M$.

(ii) For every $a \in R-(N: M)$ and every ideal $I$ of $R$ such that $I \nsubseteq(N: M)$ if $a I \nsubseteq \sqrt{(N: M)}$, then $(N: a I) \subseteq(\phi(N): a I) \cup(N: a) \cup\left(N: I^{n}\right)$ for some positive integer $n$.

(iii) For every ideals $I, J$ of $R$ such that $I, J \nsubseteq(N: M)$ if $I J \nsubseteq \sqrt{(N: M)}$, then $(N: I J) \subseteq(\phi(N): I J) \cup(N: I) \cup\left(N: J^{n}\right)$ for some positive integer $n$.

Proof. The proof is similar to Theorem 6 .

The following theorem offers a characterization of $\phi$-2-absorbing semi-primary submodules. 
Theorem 7. Let $\phi: S(M) \rightarrow S(M) \cup\{\emptyset\}$ be a function. The following conditions are equivalent:

(i) $N$ is a $\phi$-2-absorbing semi-primary submodule of $M$.

(ii) For every $a \in R-(N: M)$ and $m \in M$ if am $\notin N$, then $(N:$ am $) \subseteq(\phi(N)$ : $a m) \cup(\sqrt{((N: M)}: a) \cup \sqrt{(N: m)}$.

Proof. $(i \Rightarrow$ ii $)$ Let $a \in R-(N: M)$ and $m \in M$ such that am $\notin N$. Assume that $r \in(N: a m)$. If $\operatorname{ram} \notin \phi(N)$, then $\operatorname{ram} \in N-\phi(N)$. By Definition 1, ar $\in \sqrt{(N: M)}$ or $a m \in N$ or $r^{n} m \in N$ for some positive integer $n$. By assumption, $r \in(\sqrt{(N: M)}$ : $a) \cup \sqrt{(N: m)} \subseteq(\phi(N): a m) \cup(\sqrt{((N: M)}: a) \cup \sqrt{(N: m)}$. Now if $r a m \in \phi(N)$, then $r \in(\phi(N): a m) \subseteq(\phi(N): a m) \cup(\sqrt{((N: M)}: a) \cup \sqrt{(N: m)}$.

$(i i \Rightarrow i)$ It is obvious.

Corollary 4. Let $\phi: S(M) \rightarrow S(M) \cup\{\emptyset\}$ be a function. The following conditions are equivalent:

(i) $N$ is a $\phi$-2-absorbing semi-primary submodule of $M$.

(ii) For every ideal $I$ of $R$ such that $I \subseteq R-(N: M)$ and $m \in M$ if $I m \nsubseteq N$, then $(N: I m) \subseteq(\phi(N): I m) \cup(\sqrt{(N: M)}: I) \cup \sqrt{(N: m)}$.

Proof. The proof is similar to Theorem 7.

\section{Properties of $\phi_{\alpha}-2-$ Absorbing Semi-primary Submodules}

We start with the following theorem that gives a relation between $\phi_{\alpha}$-2-absorbing semiprimary and $\phi$-2-absorbing semi-primary submodule. Our starting points are the following definitions:

Definition 2. Let $M$ be an R-module and let $S(M)$ be the set of all submodules of $M$. Define the following functions $\phi_{\alpha}: S(M) \rightarrow S(M) \cup\{\emptyset\}$ and the corresponding $\phi_{\alpha}-2$ absorbing semi-primary submodules:

- If $\phi(N)=\emptyset$ for every $N \in \mathcal{S}(M)$, then we say that $\phi=\phi_{\emptyset}$ and $N$ is called a $\phi_{\emptyset}-2$ absorbing semi-primary submodule of $M$, and hence $N$ is a 2-absorbing semi-primary submodule of $M$.

- If $\phi(N)=\{0\}$ for every $N \in \mathcal{S}(M)$, then we say that $\phi=\phi_{0}$ and $N$ is called a $\phi_{0}$-2-absorbing semi-primary submodule of $M$, and hence $N$ is a weakly 2-absorbing semi-primary submodule of $M$.

- If $\phi(N)=N$ for every $N \in \mathcal{S}(M)$, then we say that $\phi=\phi_{1}$ and $N$ is called a $\phi_{1}$-2-absorbing semi-primary submodule of $M$. 
- If $\phi(N)=(N: M) N$ for every $N \in \mathcal{S}(M)$, then we say that $\phi=\phi_{2}$ and $N$ is called a $\phi_{2}$-2-absorbing semi-primary submodule of $M$, and hence $N$ is an almost 2-absorbing semi-primary submodule of $M$.

- If $\phi(N)=(N: M)^{n-1} N$ for every $N \in \mathcal{S}(M)$, then we say that $\phi=\phi_{n \geq 2}$ and $N$ is called a $\phi_{n}$-2-absorbing semi-primary submodule of $M$, and hence $N$ is a $n$-2absorbing semi-primary submodule of $M$.

- If $\phi(N)=\bigcap_{i=1}^{\infty}(N: M)^{i} N$ for every $N \in \mathcal{S}(M)$, then we say that $\phi=\phi_{\omega}$ and $N$ is called a $\phi_{\omega}$-2-absorbing semi-primary submodule of $M$, and hence $N$ is a $\omega-2$ absorbing semi-primary submodule of $M$.

Remark 2. Let $M$ be an $R$-module and let $S(M)$ be a set of all submodules of $M$. For two functions $\phi_{\alpha}, \phi_{\beta}: S(M) \rightarrow S(M) \cup\{\emptyset\}$. We define $\phi_{\alpha} \leq \phi_{\beta}$, if $\phi_{\alpha}(N) \subseteq \phi_{\beta}(N)$ for all $N \in S(M)$ [11]. Observe that $\phi_{\emptyset} \leq \phi_{0} \leq \phi_{\omega} \leq \ldots \leq \phi_{n+1} \leq \phi_{n} \leq \ldots \leq \phi_{2} \leq \phi_{1}$.

Notice that for an $R$-module $\mathrm{M}$, the zero submodule $\{0\}$ is always a $\phi_{0^{-}}$-2-absorbing semi-primary submodule. In the following example, we give a module in which a $\phi_{0}-2$ absorbing semi-primary submodule is not $\phi$-2-absorbing semi-primary .

Example 3. Let $R=\boldsymbol{Z}$ and $M=\boldsymbol{Z}_{30} \times \boldsymbol{Z}_{30}$. Consider the submodule $N=\{[0]\} \times \boldsymbol{Z}_{30}$ of $M$. Define $\phi: S(M) \rightarrow S(M) \cup\{\emptyset\}$ by $\phi(N)=\{[0]\} \times\{[0]\}$ for every $N \in S(M)$. It is easy to see that $N$ is a $\phi_{0}$-2-absorbing semi-primary submodule of $M$. Notice that $(2 \cdot 3)([5],[1]) \in N-\phi(N)$, but $2 \cdot 3 \notin \sqrt{(N: M)}, 2([5],[1]) \notin\{[0]\} \times \boldsymbol{Z}_{30}$ and $3^{n}([5],[1]) \notin$ $\{[0]\} \times Z_{30}$ for all positive integer $n$. Therefore $N$ is not a $\phi$-2-absorbing semi-primary submodule of $M$.

We are finding additional condition to show that a 2-absorbing semi-primary submodule is a $\phi$-2-absorbing semi-primary submodule of an $R$-module.

Theorem 8. Let $\phi: S(M) \rightarrow S(M) \cup\{\emptyset\}$ be a function and let $\phi(N)$ is a 2-absorbing semi-primary submodule of $M$. Then $N$ is a $\phi$-2-absorbing semi-primary submodule of $M$ if and only if $N$ is a 2-absorbing semi-primary submodule of $M$.

Proof. Suppose that $N$ is a 2-absorbing semi-primary submodule of $M$. Clearly, $N$ is a $\phi$-2-absorbing semi-primary submodule of $M$. Conversely, assume that $N$ is a $\phi$-2-absorbing semi-primary submodule of $M$. Let $a_{1}, a_{2} \in R$ and $m \in M$ such that $a_{1} a_{2} m \in N$. If $a_{1} a_{2} m \notin \phi(N)$, then $a_{1} a_{2} m \in N-\phi(N)$. By Definition 1, $a_{1} a_{2} \in \sqrt{(N: M)}$ or $a_{1} m \in N$ or $a_{2}^{n} m \in N$ for some positive integer $n$. Now if $a_{1} a_{2} m \in \phi(N)$, then $a_{1} a_{2} \in \sqrt{(N: M)}$ or $a_{1} m \in N$ or $a_{2}^{n} m \in N$ for some positive integer $n$.

Further, we give another characterization of $\phi_{\alpha}$-2-absorbing semi-primary submodule of $M$.

Theorem 9. Let $\phi_{2}: S(M) \rightarrow S(M) \cup\{\emptyset\}$ be a function and let $\left(0: r^{k}\right) \subseteq r^{k} M \neq M$, where $r \in R$. Then $r^{k} M$ is a $\phi_{2}$-2-absorbing semi-primary submodule of $M$ if and only if it is a 2-absorbing semi-primary submodule of $M$. 
Proof. Suppose that $r^{k} M$ is a 2-absorbing semi-primary submodule of $M$. Clearly, $r^{k} M$ is a $\phi_{2}$-2-absorbing semi-primary submodule of $M$. Conversely, assume that $r^{k} M$ is a $\phi_{2}$-2-absorbing semi-primary submodule of $M$. Let $a_{1}, a_{2} \in R$ and $m \in M$ such that $a_{1} a_{2} m \in r^{k} M$. If $a_{1} a_{2} m \notin \phi_{2}\left(r^{k} M\right)$, then $a_{1} a_{2} m \in r^{k} M-\phi_{2}\left(r^{k} M\right)$. By Definition $1, a_{1} a_{2} \in \sqrt{(N: M)}$ or $a_{1} m \in N$ or $a_{2}^{n} m \in N$ for some positive integer $n$. Assume that $a_{1} a_{2} m \in \phi_{2}\left(r^{k} M\right)$. Since $a_{1} a_{2} m, r^{k} a_{2} m \in r^{k} M$, we have $\left(a_{1}+r^{k}\right) a_{2} m \in r^{k} M$. If $\left(a_{1}+r^{k}\right) a_{2} m \notin \phi_{2}\left(r^{k} M\right)$, then $\left(a_{1}+r^{k}\right) a_{2} m \in r^{k} M-\phi_{2}\left(r^{k} M\right)$. Then by Definition 1 , $a_{1} a_{2} \in \sqrt{\left(r^{k} M: M\right)}$ or $a_{1} m \in r^{k} M$ or $a_{2}^{n} m \in r^{k} M$ for some positive integer $n$. Now if $\left(a_{1}+r^{k}\right) a_{2} m \in \phi_{2}\left(r^{k} M\right)$, then $r^{k} a_{2} m \in \phi_{2}\left(r^{k} M\right)$. Then there exists $m_{0} \in\left(r^{k} M: M\right) M$ such that $r^{k} a_{2} m=r^{k} m_{0}$. By assumption, $a_{2} m-m_{0} \in r^{k} M$. Hence $a_{2} m \in r^{k} M$.

Theorem 10. Let $\phi: S(M) \rightarrow S(M) \cup\{\emptyset\}$ be a function and let $N, K$ be two submodules of $M$ with $N \subseteq K$. If $\phi(K) \subseteq N$ and $K$ is a $\phi$-2-absorbing semi-primary submodule of $M$, then $K / N$ is a $\phi_{0}-2$-absorbing semi-primary submodule of $M / N$.

Proof. Let $a_{1}, a_{2} \in R$ and $m \in M$ such that $a_{1} a_{2} m+N \in K / N-\left(\phi_{N}\right)_{0}(K / N)$. Since $\phi(K) \subseteq N$, we have $a_{1} a_{2} m \notin \phi(K)$. Clearly, $a_{1} a_{2} m \in K-\phi(K)$. By Definition $1, a_{1} a_{2} \in \sqrt{(K: M)}$ or $a_{1} m \in K$ or $a_{2}^{n} m \in K$ for some positive integer $n$. Thus $a_{1} a_{2} \in$ $\sqrt{(K / N: M / N)}$ or $a_{1}(m+N) \in K / N$ or $a_{2}^{n}(m+N) \in K / N$ for some positive integer $n$.

Theorem 11. Let $\phi: S(M) \rightarrow S(M) \cup\{\emptyset\}$ be a function, $N$ be a $\phi$-2-absorbing semiprimary submodule of $M$ and let $K$ be a submodule of $M$ with $N \subseteq K$. If $\phi(N) \subseteq \phi(K)$ and $K / N$ is a $\phi_{0}$-2-absorbing semi-primary submodule of $M / N$, then $K$ is a $\phi$-2-absorbing semi-primary submodule of $M$.

Proof. Let $a_{1}, a_{2} \in R$ and $m \in M$ such that $a_{1} a_{2} m \in K-\phi(K)$. By assumption, $a_{1} a_{2} m \notin \phi(N)$. If $a_{1} a_{2} m \in N$, then $a_{1} a_{2} m \in N-\phi(N)$. By Definition 1, $a_{1} a_{2} \in$ $\sqrt{(N: M)} \subseteq \sqrt{(K: M)}$ or $a_{1} m \in N \subseteq K$ or $a_{2}^{n} m \in N \subseteq K$ for some positive integer $n$. If $a_{1} a_{2} m \notin N$, then $a_{1} a_{2}(m+N) \notin \phi_{0}(K / N)$. Therefore $a_{1} a_{2}(m+N) \in K / N-\phi_{0}(K / N)$. By Definition $1, a_{1} a_{2} \in \sqrt{(K / N: M / N)}$ or $a_{1}(m+N) \in K / N$ or $a_{2}^{n}(m+N) \in K / N$ for some positive integer $n$. This completes the proof.

As an immediate consequence of Theorem 10 and Theorem 11 we have the next corollary.

Corollary 5. Let $\phi: S(M) \rightarrow S(M) \cup\{\emptyset\}$ be a function and let $N, K$ be two submodules of $M$ with $N \subseteq K$. Then $N$ is a $\phi$-2-absorbing semi-primary submodule of $M$ if and only if $N / \phi(N)$ is a $\phi_{0}$-2-absorbing semi-primary submodule of $M / \phi(N)$.

Proof. It is straightforward by Theorem 10 and Theorem 11.

Theorem 12. Let $\phi_{\alpha}: S(M) \rightarrow S(M) \cup\{\emptyset\}$ be a function. Then the following hold.

(i) If $N$ is a $\phi_{\beta}$-2-absorbing semi-primary submodule of $M$ such that $\phi_{\beta} \leq \phi_{\gamma}$, then $N$ is a $\phi_{\gamma}$-2-absorbing semi-primary submodule of $M$. 
(ii) If $N$ is a $\phi_{\emptyset}$-2-absorbing semi-primary submodule of $M$, then $N$ is a $\phi_{0}$-2-absorbing semi-primary submodule of $M$.

(iii) If $N$ is a $\phi_{0}$-2-absorbing semi-primary submodule of $M$, then $N$ is an $\omega$-2-absorbing semi-primary submodule of $M$.

(iv) If $N$ is a $\phi_{\omega}$-2-absorbing semi-primary submodule of $M$, then $N$ is a $\phi_{n}$-2-absorbing semi-primary submodule of $M$.

Proof. i. Let $a_{1}, a_{2} \in R$ and $m \in M$ such that $a_{1} a_{2} m \in N-\phi_{\gamma}(N)$. By assumption, $\phi_{\beta}(N) \subseteq \phi_{\gamma}(N)$. Then $a_{1} a_{2} m \in N-\phi_{\gamma}(N) \subseteq N-\phi_{\beta}(N)$. Then by Definition 1, $a_{1} a_{2} \in \sqrt{(N: M)}$ or $a_{1} m \in N$ or $a_{2}^{n} m \in N$ for some positive integer $n$.

ii, iii, iv. It is obvious.

From the above definitions we obtain immediately the following implication chart for the considered types of submodules:

2-absorbing semi-primary $\Rightarrow$ weakly 2 -absorbing semi-primary $\Rightarrow \omega$-2-absorbing semi-primary $\Rightarrow \phi_{n \geq 2}$-2-absorbing semi-primary $\Rightarrow$ almost 2-absorbing semi-primary

Theorem 13. Let $\phi, \phi_{3}: S(M) \rightarrow S(M) \cup\{\emptyset\}$ be two functions and let $N$ be a $\phi-2$ absorbing semi-primary submodule. If $\phi_{3} \not \leq \phi$, then $N$ is a 2-absorbing semi-primary submodule of $M$.

Proof. Let $a_{1}, a_{2} \in R$ and $m \in M$ such that $a_{1} a_{2} m \in N$. If $a_{1} a_{2} m \notin \phi(N)$, then $a_{1} a_{2} m \in N-\phi(N)$. By Definition $1, a_{1} a_{2} \in \sqrt{(N: M)}$ or $a_{1} m \in N$ or $a_{2}^{n} m \in N$ for some positive integer $n$. Next, let $a_{1} a_{2} m \in \phi(N)$. In this case, we may assume that $a_{1} a_{2} N \subseteq \phi(N)$, because if $a_{1} a_{2} N \nsubseteq \phi(N)$ then there exists $m_{0} \in N$ such that $a_{1} a_{2} m_{0} \notin \phi(N)$. Clearly, $a_{1} m \in N$ or $a_{1} a_{2} \in \sqrt{(N: M)}$ or $a_{2}^{n} m \in N$ for some positive integer $n$. Second we may assume that $(N: M)^{2} m \subseteq \phi(N)$. If this is not the case, there exist $r_{1}, r_{2} \in(N: M)$ such that $\left(a_{1}+r_{1}\right)\left(a_{2}+r_{2}\right) m \notin \phi(N)$. By assumption, $a_{1} m \in N$ or $a_{1} a_{2} \in \sqrt{(N: M)}$ or $a_{2}^{n} \in \sqrt{(N: m)}$ for some positive integer $n$. Again, by assumption, $(N: M)^{2} N \nsubseteq \phi(N)$. There exist $r_{1}, r_{2} \in(N: M)$ and $m_{0} \in N$ such that $r_{1} r_{2} m_{0} \notin \phi(N)$. Thus by Definition $1, a_{1} m \in N$ or $a_{1} a_{2} \in \sqrt{(N: M)}$ or $a_{2}^{n} m \in N$ for some positive integer $n$.

Corollary 6. Let $\phi_{n}: S(M) \rightarrow S(M) \cup\{\emptyset\}$ be a function and let $N$ be a $\phi_{0}$-2-absorbing semi-primary submodule of $M$. If $\phi_{3} \neq \phi_{0}$, then $N$ is a 2-absorbing semi-primary submodule of $M$.

Proof. Similar to the proof of Theorem 13.

Theorem 14. Let $\phi, \phi_{4}: S(M) \rightarrow S(M) \cup\{\emptyset\}$ be two functions. If $N$ is a $\phi$-2-absorbing semi-primary submodule such that $\phi \leq \phi_{4}$, then $N$ is a $\omega$-2-absorbing semi-primary submodule of $M$. 
Proof. If $N$ is a 2-absorbing semi-primary submodule of $M$, then there is nothing to prove. Assume that $N$ is not a 2-absorbing semi-primary submodule of $M$. Then by Theorem 13, $(N: M)^{2} N=\phi_{3}(N) \subseteq \phi(N) \subseteq(N: M)^{3} N$. This implies that $\phi(N)=(N$ : $M)^{2} N=(N: M)^{3} N$. Thus $\phi(N)=(N: M)^{i} N$ for all $i \geq 3$.

Theorem 15. Let $M$ be a multiplication $R$-module and $\phi: S(M) \rightarrow S(M) \cup\{\emptyset\}$ be a function. Then the following properties hold.

(i) If $N$ is a $\phi$-2-absorbing semi-primary submodule of $M$ with $N^{3} \nsubseteq \phi(N)$, then $N$ is a 2-absorbing semi-primary submodule of $M$.

(ii) If $N$ is a $\phi_{n}$-2-absorbing semi-primary submodule of $M$ with $N^{3} \neq N^{n}$ for all $n \geq 3$, then $N$ is a 2-absorbing semi-primary submodule of $M$.

Proof. 1. Suppose that $N$ is a $\phi$-2-absorbing semi-primary submodule of $M$ that is not 2-absorbing semi-primary. Clearly, $N=(N: M) M$. Then by Theorem $13, N^{3}=(N$ : $M)^{3} M=(N: M)^{2}((N: M) M)=(N: M)^{2} N=\phi_{3}(N) \subseteq \phi(N)$.

2. Suppose that $N$ is a $\phi_{n}$-2-absorbing semi-primary submodule of $M$ that is not 2absorbing semi-primary. Clearly, $N^{n} \subseteq N^{3}$, for all $n \geq 3$. Then by parts $1, N^{3} \subseteq \phi_{n}(N)=$ $(N: M)^{n-1} N=(N: M)^{n} M=N^{n}$. Hence $N^{3}=N^{n}$. This completes the proof.

Theorem 16. Let $\psi_{i}: S\left(M_{i}\right) \rightarrow S\left(M_{i}\right) \cup\{\emptyset\}$ be a function with $\phi=\psi_{1} \times \psi_{2}$. Then the following statements are equivalent:

(i) $N_{1} \times M_{2}$ is a $\phi$-2-absorbing semi-primary submodule of $M_{1} \times M_{2}$.

(ii) (a) $N_{1}$ is a $\psi_{1}$-2-absorbing semi-primary submodule of $M_{1}$.

(b) For each $a_{1}, a_{2} \in R$ and $m \in M_{1}$ such that $a_{1} a_{2} m \in \psi_{1}\left(N_{1}\right)$ if $a_{1} a_{2} \notin$ $\sqrt{\left(N_{1}: M_{1}\right)}$ and $a_{1} m \notin N_{1}, a_{2}^{n} m \notin N_{1}$ for all positive integer $n$, then $a_{1} a_{2} \in$ $\left(\psi_{2}\left(M_{2}\right): M_{2}\right)$.

Proof. $(i \Rightarrow i i)$. (a). It is obvious.

(b). Let $a_{1} a_{2} m \in \psi_{1}\left(N_{1}\right), a_{1} m \notin N_{1}$ and $a_{2}^{n} m \notin N_{1}$, where $a_{1}, a_{2} \in R$ and $m \in M_{1}$. Suppose that $a_{1} a_{2} \notin\left(\psi_{2}\left(M_{2}\right): M_{2}\right)$. There exists $m_{2} \in M_{2}$ such that $a_{1} a_{2} m_{2} \notin \psi_{2}\left(M_{2}\right)$. Thus $a_{1} a_{2}\left(m, m_{2}\right) \in N_{1} \times M_{2}-\phi\left(N_{1} \times M_{2}\right)$. By part (1), i.e., $a_{1} a_{2} \in \sqrt{\left(N_{1}: M_{1}\right)}$ or $a_{1} m \in N_{1}$ or $a_{2}^{n} m \in N_{1}$ which is a contradiction.

$(i i \Rightarrow i)$. Let $a_{1}, a_{2} \in R$ and $\left(m_{1}, m_{2}\right) \in M_{1} \times M_{2}$ such that $a_{1} a_{2}\left(m_{1}, m_{2}\right) \in N_{1} \times$ $M_{2}-\phi\left(N_{1} \times M_{2}\right)$. If $a_{1} a_{2} m_{1} \notin \psi_{1}\left(N_{1}\right)$, then $a_{1} a_{2} m_{1} \in N_{1}-\psi_{1}\left(N_{1}\right)$. By part (a), $a_{1} a_{2} \in \sqrt{\left(N_{1} \times M_{2}: M_{1} \times M_{2}\right)}$ or $a_{1}\left(m_{1}, m_{2}\right)=\left(a_{1} m_{1}, a_{1} m_{2}\right) \in N_{1} \times M_{2}$ or $a_{2}^{n}\left(m_{1}, m_{2}\right)=$ $\left(a_{2}^{n} m_{1}, a_{2}^{n} m_{2}\right) \in N_{1} \times M_{2}$, and thus we are done. If $a_{1} a_{2} m_{1} \in \psi_{1}\left(N_{1}\right)$, then $a_{1} a_{2} m_{2} \notin$ $\psi_{2}\left(M_{2}\right)$. Therefore $a_{1} a_{2} \notin\left(\psi_{2}\left(M_{2}\right): M_{2}\right)$. By part (b), $a_{1} a_{2} \in \sqrt{\left(N_{1} \times M_{2}: M_{1} \times M_{2}\right)}$ or $a_{1}\left(m_{1}, m_{2}\right) \in N_{1} \times M_{2}$ or $a_{2}^{n}\left(m_{1}, m_{2}\right) \in N_{1} \times M_{2}$.

Corollary 7. Let $\psi_{i}: S\left(M_{i}\right) \rightarrow S\left(M_{i}\right) \cup\{\emptyset\}$ be a function with $\phi=\psi_{1} \times \psi_{2}$. Then the following conditions are equivalent: 
(i) $M_{1} \times N_{2}$ is a $\phi$-2-absorbing semi-primary submodule of $M_{1} \times M_{2}$.

(ii) (a) $N_{2}$ is a $\psi_{2}$-2-absorbing semi-primary submodule of $M_{2}$.

(b) For each $a_{1}, a_{2} \in R$ and $m \in M_{2}$ such that $a_{1} a_{2} m \in \psi_{2}\left(N_{2}\right)$, if $a_{1} a_{2} \notin$ $\sqrt{\left(N_{2}: M_{2}\right)}, a_{1} m \notin N_{2}$ and $a_{2}^{n} m \notin N_{2}$ for all positive integer $n$, then $a_{1} a_{2} \in$ $\left(\psi_{1}\left(M_{1}\right): M_{1}\right)$.

Proof. Similar to the proof of Theorem 16.

Theorem 17. Let $\psi_{i}: S\left(M_{i}\right) \rightarrow S\left(M_{i}\right) \cup\{\emptyset\}$ is a function with $\phi=\psi_{1} \times \ldots \times \psi_{k}$. Then the following conditions are equivalent:

(i) $M_{1} \times \ldots \times M_{i-1} \times N_{i} \times M_{i+1} \times \ldots \times M_{k}$ is a $\phi$-2-absorbing semi-primary submodule of $M_{1} \times \ldots \times M_{k}$.

(ii) (a) $N_{i}$ is a $\psi_{i}$-2-absorbing semi-primary submodule of $M_{i}$.

(b) For each $a_{1}, a_{2} \in R$ and $m \in M_{i}$ such that $a_{1} a_{2} m \in \psi_{i}\left(N_{i}\right)$, if $a_{1} a_{2} \notin$ $\sqrt{\left(N_{i}: M_{i}\right)}, a_{1} m \notin N_{i}$ and $a_{2}^{n} m \notin N_{i}$ for all positive integer $n$, then there exists $j \in\{1,2, \ldots, k\}$ such that $a_{1} a_{2} \in\left(\psi_{j}\left(M_{j}\right): M_{j}\right)$.

Proof. Similar to the proof of Theorem 16.

Next, let $R_{i}$ be a commutative ring with identity and let $M_{i}$ be an $R_{i}$-module. Then $M_{1} \times M_{2}$ is an $R_{1} \times R_{2}$-module and each submodule of $M_{1} \times M_{2}$ is of the form $N_{1} \times N_{2}$ for some submodules $N_{1}$ of $M_{1}$ and $N_{2}$ of $M_{2}$. Next we show that, if $N_{1}$ is a $\left(\psi_{1}\right)_{0}$-2-absorbing semi-primary submodule of $M_{1}$, then $N_{1} \times M_{2}$ is a $\phi$-2-absorbing semi-primary submodule if $\{0\} \times M_{2} \subseteq \psi_{1} \times \psi_{2}\left(N_{1} \times M_{2}\right)$. First, we would like to show that, $N_{1}$ is a $\psi_{1}$-2-absorbing semi-primary submodule of $M_{1}$ if $N_{1} \times M_{2}$ is a $\phi$-2-absorbing semi-primary submodule of $M_{1} \times M_{2}$.

Theorem 18. Let $\psi_{i}: S\left(M_{i}\right) \rightarrow S\left(M_{i}\right) \cup\{\emptyset\}$ be a function with $\phi=\psi_{1} \times \psi_{2}$. If $N_{1} \times M_{2}$ is a $\phi$-2-absorbing semi-primary submodule of $M_{1} \times M_{2}$, then $N_{1}$ is a $\psi_{1}$-2-absorbing semi-primary submodule of $M_{1}$.

Proof. Let $a_{1}, a_{2} \in R_{1}$ and $m \in M_{1}$ such that $a_{1} a_{2} m \in N_{1}-\psi_{1}\left(N_{1}\right)$. Then $\left(a_{1}, 0\right)\left(a_{2}, 0\right)(m, 0) \in N_{1} \times M_{2}-\psi_{1}\left(N_{1}\right) \times \psi_{2}\left(M_{2}\right)$. By Definition 1, $a_{1} a_{2} \in \sqrt{\left(N_{1}: M_{1}\right)}$ or $a_{1} m \in N_{1}$ or $a_{2}^{n} m \in N_{1}$.

Lemma 1. Let $\psi_{i}: S\left(M_{i}\right) \rightarrow S\left(M_{i}\right) \cup\{\emptyset\}$ be a function. If $N_{1}$ is a $\left(\psi_{1}\right)_{0}$-2-absorbing semi-primary submodule of $M_{1}$ such that $\{0\} \times M_{2} \subseteq \psi_{1} \times \psi_{2}\left(N_{1} \times M_{2}\right)$, then $N_{1} \times M_{2}$ is a $\psi_{1} \times \psi_{2}$-2-absorbing semi-primary submodule of $M_{1} \times M_{2}$.

Proof. Let $\left(a_{1}, b_{1}\right),\left(a_{2}, b_{2}\right) \in R_{1} \times R_{2}$ and $\left(m_{1}, m_{2}\right) \in M_{1} \times M_{2}$ such that

$$
\left(a_{1}, b_{1}\right)\left(a_{2}, b_{2}\right)\left(m_{1}, m_{2}\right) \in N_{1} \times M_{2}-\phi\left(N_{1} \times M_{2}\right) .
$$


By assumption, $\left(a_{1} a_{2} m_{1}, b_{1} b_{2} m_{2}\right) \notin\{0\} \times M_{2}$. Clearly, $a_{1} a_{2} m_{1} \in N_{1}-\left(\psi_{1}\right)_{0}\left(N_{1}\right)$. By Definition $1, a_{1} a_{2} \in \sqrt{\left(N_{1}: M_{1}\right)}$ or $a_{1} m_{1} \in N_{1}$ or $a_{2}^{n} m_{1} \in N_{1}$ for some positive integer $n$. Therefore $N_{1} \times M_{2}$ is a $\phi$-2-absorbing semi-primary submodule of $M_{1} \times M_{2}$.

Corollary 8. Let $\psi_{i}: S\left(M_{i}\right) \rightarrow S\left(M_{i}\right) \cup\{\emptyset\}$ be a function with $\phi=\psi_{1} \times \psi_{2}$. If $N_{2}$ is a $\left(\psi_{2}\right)_{0}$-2-absorbing semi-primary submodule of $M_{2}$ such that $M_{1} \times\{0\} \subseteq \psi_{1} \times \psi_{2}\left(M_{1} \times N_{2}\right)$, then $M_{1} \times N_{2}$ is a $\phi$-2-absorbing semi-primary submodule of $M_{1} \times M_{2}$.

Proof. Similar to the proof of Lemma 1.

Theorem 19. Let $\psi_{i}: S\left(M_{i}\right) \rightarrow S\left(M_{i}\right) \cup\{\emptyset\}$ be a function with $\phi=\psi_{1} \times \ldots \times \psi_{k}$ and $M_{1} \times \ldots \times M_{i-1} \times\{0\} \times M_{i+1} \times \ldots \times M_{k} \subseteq \phi\left(M_{1} \times \ldots \times M_{i-1} \times N_{i} \times M_{i+1} \times\right.$ $\left.\ldots \times M_{k}\right)$. Then $N_{j}$ is a $\left(\psi_{j}\right)_{0}$-2-absorbing semi-primary submodule of $M_{j}$ if and only if $M_{1} \times M_{2} \times \ldots \times M_{i-1} \times N_{i} \times M_{i+1} \times \ldots \times M_{k}$ is a $\phi$-2-absorbing semi-primary submodule of $M_{1} \times M_{2} \times \ldots \times M_{k}$.

Proof. Similar to the proof of Theorem 18 and Lemma 1.

Theorem 20. Let $\left(\psi_{i}\right)_{n}: S\left(M_{i}\right) \rightarrow S\left(M_{i}\right) \cup\{\emptyset\}$ be a function with $\phi_{n}=\left(\psi_{1}\right)_{n} \times\left(\psi_{2}\right)_{n}$. If $N_{1}$ is a $\left(\psi_{1}\right)_{0}$-2-absorbing semi-primary submodule of $M_{1}$ such that $\left(\psi_{2}\right)_{3}\left(M_{2}\right)=M_{2}$, then $N_{1} \times M_{2}$ is a $\phi_{3}$-2-absorbing semi-primary submodule of $M_{1} \times M_{2}$.

Proof. If $N_{1}$ is a 2-absorbing semi-primary submodule of $M_{1}$, then $N_{1} \times M_{2}$ is a 2absorbing semi-primary submodule of $M_{1} \times M_{2}$. Clearly, $N_{1} \times M_{2}$ is a $\phi_{3}$-2-absorbing semi-primary submodule of $M_{1} \times M_{2}$. Assume that $N_{1}$ is not 2-absorbing semi-primary. By Corollary 6, $\left(\psi_{1}\right)_{3} \leq\left(\psi_{1}\right)_{0}$ so $\left(N_{1}: M_{1}\right)^{2} N_{1}=\{0\}$. Therefore $\left(\psi_{1}\right)_{3} \times\left(\psi_{2}\right)_{3}\left(N_{1} \times M_{2}\right)=$ $\left(\psi_{1}\right)_{3}\left(N_{1}\right) \times\left(\psi_{2}\right)_{3}\left(M_{2}\right)=\{0\} \times M_{2}$. Now, by Lemma $1, N_{1} \times M_{2}$ is a $\left(\psi_{1}\right)_{3} \times\left(\psi_{2}\right)_{3}-2-$ absorbing semi-primary submodule of $M_{1} \times M_{2}$.

Corollary 9. Let $\left(\psi_{i}\right)_{n}: S\left(M_{i}\right) \rightarrow S\left(M_{i}\right) \cup\{\emptyset\}$ be a function with $\phi_{n}=\left(\psi_{1}\right)_{n} \times\left(\psi_{2}\right)_{n}$. If $N_{2}$ is a $\left(\psi_{2}\right)_{0}$-2-absorbing semi-primary submodule of $M_{2}$ such that $\left(\psi_{1}\right)_{3}\left(M_{1}\right)=M_{1}$, then $M_{1} \times N_{2}$ is a $\phi_{3}$-2-absorbing semi-primary submodule of $M_{1} \times M_{2}$.

Proof. Similar to the proof of Theorem 20.

Theorem 21. Let $\left(\psi_{i}\right)_{n}: S\left(M_{i}\right) \rightarrow S\left(M_{i}\right) \cup\{\emptyset\}$ be a function with $\phi_{n}=\left(\psi_{1}\right)_{n} \times \ldots \times\left(\psi_{k}\right)_{n}$. If $N_{j}$ is a $\left(\psi_{j}\right)_{0}$-2-absorbing semi-primary submodule of $M_{j}$ such that $\left(\psi_{j}\right)_{3}\left(M_{j}\right)=M_{j}$, then $M_{1} \times M_{2} \times \ldots \times M_{i-1} \times N_{i} \times M_{i+1} \times \ldots \times M_{k}$ is a $\phi_{3}$-2-absorbing semi-primary submodule of $M_{1} \times \ldots \times M_{k}$.

Proof. Similar to the proof of Theorem 20 and Corollary 9.

Theorem 22. Let $\psi_{i}: S\left(M_{i}\right) \rightarrow S\left(M_{i}\right) \cup\{\emptyset\}$ be a function with $\phi=\psi_{1} \times \psi_{2}$. Then the following conditions are equivalent: 
(i) $N_{1}$ is a 2-absorbing semi-primary submodule of $M_{1}$.

(ii) $N_{1} \times M_{2}$ is a 2-absorbing semi-primary submodule of $M_{1} \times M_{2}$.

(iii) $N_{1} \times M_{2}$ is a $\phi$-2-absorbing semi-primary submodule of $M_{1} \times M_{2}$, where $\psi_{2}\left(M_{2}\right) \neq$ $\mathrm{M}_{2}$.

Proof. $(1 \Rightarrow 2)$. Let $\left(a_{1}, b_{1}\right),\left(a_{2}, b_{2}\right) \in R_{1} \times R_{2}$ and $\left(m_{1}, m_{2}\right) \in M_{1} \times M_{2}$ such that $\left(a_{1}, b_{1}\right)\left(a_{2}, b_{2}\right)\left(m_{1}, m_{2}\right) \in N_{1} \times M_{2}$. Clearly, $a_{1} a_{2} m_{1} \in N_{1}$. By Definition $2, a_{1} a_{2} \in$ $\sqrt{\left(N_{1}: M_{1}\right)}$ or $a_{1} m_{1} \in N_{1}$ or $a_{2}^{n} m_{1} \in N_{1}$ for some positive integer $n$. This completes the proof.

$(2 \Rightarrow 3)$. It is easy to see that every 2 -absorbing primary submodule is $\phi$-2-absorbing semi-primary.

$(3 \Rightarrow 1)$. Let $a_{1}, a_{2} \in R_{1}$ and $m \in M_{1}$ such that $a_{1} a_{2} m \in N_{1}$. By assumption, there exists $m_{2} \in M_{2}$ such that $m_{2} \notin \psi_{2}\left(M_{2}\right)$. Since $\phi\left(N_{1} \times M_{2}\right) \subseteq M_{1} \times \psi_{2}\left(M_{2}\right)$, we have $\left(a_{1}, 1\right)\left(a_{2}, 1\right)\left(m, m_{2}\right) \in N_{1} \times M_{2}-\psi_{1} \times \psi_{2}\left(N_{1} \times M_{2}\right)$. By Definition 1, $a_{1} a_{2} \in \sqrt{\left(N_{1}: M_{1}\right)}$ or $a_{1} m \in N_{1}$ or $a_{2}^{n} m \in N_{1}$.

Corollary 10. Let $\psi_{i}: S\left(M_{i}\right) \rightarrow S\left(M_{i}\right) \cup\{\emptyset\}$ be a function $\phi=\psi_{1} \times \psi_{2}$. Then the following conditions are equivalent:

(i) $\mathrm{N}_{2}$ is a 2-absorbing semi-primary submodule of $M_{2}$.

(ii) $M_{1} \times N_{2}$ is a 2-absorbing semi-primary submodule of $M_{1} \times M_{2}$.

(iii) $M_{1} \times N_{2}$ is a $\phi$-2-absorbing semi-primary submodule of $M_{1} \times M_{2}$, where $\psi_{1}\left(M_{1}\right) \neq$ $M_{1}$.

Proof. Similar to the proof of Theorem 22.

Theorem 23. Let $\psi_{i}: S\left(M_{i}\right) \rightarrow S\left(M_{i}\right) \cup\{\emptyset\}$ be a function with $\phi=\psi_{1} \times \ldots \times \psi_{k}$. Then the following conditions are equivalent:

(i) $N_{i}$ is a 2-absorbing semi-primary submodule of $M_{i}$.

(ii) $M_{1} \times M_{2} \times \ldots \times M_{i-1} \times N_{i} \times M_{i+1} \times \ldots \times M_{k}$ is a 2-absorbing semi-primary submodule of $M_{1} \times \ldots \times M_{2}$.

(iii) $M_{1} \times M_{2} \times \ldots \times M_{i-1} \times N_{i} \times M_{i+1} \times \ldots \times M_{k}$ is a $\phi$-2-absorbing semi-primary submodule of $M_{1} \times \ldots \times M_{2}$ with $\psi_{j}\left(M_{j}\right) \neq M_{j}$.

Proof. Similar to the proof of Theorem 22 and Corollary 10.

Theorem 24. Let $\psi_{i}: S\left(M_{i}\right) \rightarrow S\left(M_{i}\right) \cup\{\emptyset\}$ be a function with $\psi_{2}\left(M_{2}\right)=M_{2}$ and $\phi=\psi_{1} \times \psi_{2}$. Then $N_{1} \times M_{2}$ is a $\phi$-2-absorbing semi-primary submodule of $M_{1} \times M_{2}$ if and only if $N_{1}$ is a $\psi_{1}$-2-absorbing semi-primary submodule of $M_{1}$. 
Proof. The proof is clear.

Corollary 11. Let $\psi_{i}: S\left(M_{i}\right) \rightarrow S\left(M_{i}\right) \cup\{\emptyset\}$ be a function with $\psi_{1}\left(M_{1}\right)=M_{1}$ and $\phi=\psi_{1} \times \psi_{2}$. Then $M_{1} \times N_{2}$ is a $\phi$-2-absorbing semi-primary submodule of $M_{1} \times M_{2}$ if and only if $N_{2}$ is a $\psi_{2}$-2-absorbing semi-primary submodule of $M_{2}$.

Proof. Similar to the proof of Theorem 24.

Theorem 25. Let $\psi_{i}: S\left(M_{i}\right) \rightarrow S\left(M_{i}\right) \cup\{\emptyset\}$ be a function with $\psi_{j}\left(M_{j}\right)=M_{j}$ and $\phi=\psi_{1} \times \ldots \times \psi_{k}$. Then $M_{1} \times M_{2} \times \ldots \times M_{i-1} \times N_{i} \times M_{i+1} \times \ldots \times M_{k}$ is a $\phi-2$ absorbing semi-primary submodule of $M_{1} \times \ldots \times M_{k}$ if and only if $N_{i}$ is a $\psi_{i}$-2-absorbing semi-primary submodule of $M_{i}$.

Proof. Similar to the proof of Theorem 24 and Corollary 11.

Theorem 26. Let $N_{i}$ be a proper submodule of $M_{i}$ and let $\psi_{i}: S\left(M_{i}\right) \rightarrow S\left(M_{i}\right) \cup\{\emptyset\}$ be a function with $\phi=\psi_{1} \times \psi_{2}$. If $N_{1} \times N_{2}$ is a $\phi$-2-absorbing semi-primary submodule of $M_{1} \times M_{2}$, then

(i) $N_{1}$ is a $\psi_{1}$-2-absorbing semi-primary submodule of $M_{1}$,

(ii) $\mathrm{N}_{2}$ is a $\psi_{2}$-2-absorbing semi-primary submodule of $M_{2}$.

Proof. The proof is clear.

The next theorem gives conditions for a $\phi$-2-absorbing semi-primary to be 2 -absorbing semi-primary.

Theorem 27. Let $\psi_{i}: S\left(M_{i}\right) \rightarrow S\left(M_{i}\right) \cup\{\emptyset\}$ be a function with $\psi_{i}\left(M_{i}\right) \neq M_{i}, \phi=$ $\psi_{1} \times \psi_{2} \times \psi_{3}$. If $N$ is a $\phi$-2-absorbing semi-primary submodule of $M_{1} \times M_{2} \times M_{3}$, then $N=\phi(N)$ or $N$ is a 2-absorbing semi-primary submodule of $M_{1} \times M_{2} \times M_{3}$.

Proof. Suppose that $N$ is a $\phi$-2-absorbing semi-primary submodule of $M_{1} \times M_{2} \times$ $M_{3}$ that is not 2-absorbing semi-primary. Now suppose that $N_{1} \times N_{2} \times N_{3}=N \neq$ $\psi_{1} \times \psi_{2} \times \psi_{3}(N)$. Thus $N_{i} \neq \psi_{i}\left(N_{i}\right)$ for some $i=1,2,3$. We may assume that $N_{1} \neq \psi_{1}\left(N_{1}\right)$. There exists $m_{1} \in N_{1}$ such that $m_{1} \notin \psi_{1}\left(N_{1}\right)$. Assume that $N_{2} \neq M_{2}$ and $N_{3} \neq M_{3}$. Thus there exist $m_{2} \in M_{2}$ and $m_{3} \in M_{3}$ such that $m_{2} \notin N_{2}$ and $m_{3} \notin N_{3}$. Since $(1,0,1)(1,1,0)\left(m_{1}, m_{2}, m_{3}\right) \notin \psi_{1} \times \psi_{2} \times \psi_{3}\left(N_{1} \times N_{2} \times N_{3}\right)$, we have $(1,0,1)(1,1,0)\left(m_{1}, m_{2}, m_{3}\right) \in N-\phi(N)$. By Definition $1, m_{2} \in N_{2}$ or $m_{3} \in N_{3}$, a contradiction. Therefore $N=N_{1} \times M_{2} \times N_{3}$ or $N=N_{1} \times N_{2} \times M_{3}$. If $N=N_{1} \times M_{2} \times N_{3}$, then $(0,1,0) \in\left(N: M_{1} \times M_{2} \times M_{3}\right)$. By Theorem 13, $\{0\} \times M_{2} \times\{0\}=(0,1,0)^{2} N \subseteq\left(N: N_{1} \times\right.$ $\left.M_{2} \times N_{3}\right)^{2} N=\left(\psi_{1}\right)_{3} \times\left(\psi_{2}\right)_{3} \times\left(\psi_{3}\right)_{3}(N) \subseteq \psi_{1} \times \psi_{2} \times \psi_{3}(N)=\psi_{1}\left(N_{1}\right) \times \psi_{2}\left(M_{2}\right) \times \psi_{3}\left(N_{3}\right)$, which is a contradiction. This completes the proof.

The above theorem shows the relationship between 2-absorbing semi-primary and $\phi-2$ absorbing semi-primary submodules in $R_{1} \times R_{2} \times R_{3}$-modules. From the above theorem, we have the following corollary. 
Corollary 12. Let $\psi_{i}: S\left(M_{i}\right) \rightarrow S\left(M_{i}\right) \cup\{\emptyset\}$ be a function with $\psi_{i}\left(M_{i}\right) \neq M_{i}, \phi=$ $\psi_{1} \times \psi_{2} \times \psi_{3}$ and $N \neq \phi(N)$. Then $N$ is a $\phi$-2-absorbing semi-primary submodule of $M_{1} \times M_{2} \times M_{3}$ if and only if $N$ is a 2-absorbing semi-primary submodule of $M_{1} \times M_{2} \times M_{3}$.

Proof. This follows from Theorem 27.

\section{References}

[1] E A Ugurlu G Ulucak A Badawi, U Tekir and E Y Celikel. Generalizations of 2absorbing primary ideals of commutative rings. TURKISH JOURNAL OF MATHEMATICS, 40:703 - 717, 2016.

[2] D D Anderson and M Batanieh. Generalizations of prime ideals. Communications in Algebra, 36:686 - 696, 2008.

[3] D D Anderson and E. Smith. Weakly prime ideals. Houston Journal of Mathematics, 29:831 - 840, 2003.

[4] D F Anderson and A Badawi. On $n$-absorbing ideals of commutative rings. Communications in Algebra, 39(5):1646 - 1672, 2011.

[5] A Badawi. On 2-absorbing ideals of commutative rings. Bulletin of the Australian Mathematical Society, 75:417 - 429, 2007.

[6] A Badawi and A Y Darani. On weakly 2-absorbing ideals of commutative rings. Houston Journal of Mathematics, 39:441 - 452, 2013.

[7] A Y Darani. Generalizations of primary ideals in commutative rings. Novi Sad Journal of Mathematics, 42(1):27-35, 2012.

[8] M Ebrahimpour and F Mirzaee. On $\phi$-semiprime submodules. Journal of the Korean Mathematical Society, 54(4):1099 - 1108, 2017.

[9] M Ebrahimpour and R Nekooei. On generalizations of prime ideals. Communications in Algebra, 40:1268 - 1279, 2012.

[10] R Moradi and M Ebrahimpour. On $\phi$-2-absorbing primary submodules. Acta Mathematica Vietnamica, 42:27 - 35, 2017.

[11] N Zamani. $\phi$-prime submodules. Glasgow Mathematical Journal, 52(2):253 - 259, 2010. 